\title{
A population pharmacokinetic model of piperaquine in pregnant and non-pregnant women with uncomplicated Plasmodium falciparum malaria in Sudan
}

Richard M Hoglund', Ishag Adam², Warunee Hanpithakpong ${ }^{3}$, Michael Ashton ${ }^{1}$, Niklas Lindegardh ${ }^{3,4^{\wedge}}$, Nicholas PJ Day ${ }^{3,4}$, Nicholas J White ${ }^{3,4}$, Francois Nosten ${ }^{3,4,5}$ and Joel Tarning ${ }^{3,4^{*}}$

\begin{abstract}
Background: Pregnancy is associated with an increased risk of developing a malaria infection and a higher risk of developing severe malaria. The pharmacokinetic properties of many anti-malarials are also altered during pregnancy, often resulting in a decreased drug exposure. Piperaquine is a promising anti-malarial partner drug used in a fixed-dose combination with dihydroartemisinin. The aim of this study was to investigate the population pharmacokinetics of piperaquine in pregnant and non-pregnant Sudanese women with uncomplicated Plasmodium falciparum malaria.

Method: Symptomatic patients received a standard dose regimen of the fixed dose oral piperaquine-dihydroartemisinin combination treatment. Densely sampled plasma aliquots were collected and analysed using a previously described LC-MS/MS method. Data from 12 pregnant and 12 non-pregnant women were analysed using nonlinear mixed-effects modelling. A Monte Carlo Mapped Power (MCMP) analysis was conducted based on a previously published study to evaluate the power of detecting covariates in this relatively small study.

Results: A three-compartment disposition model with a transit-absorption model described the observed data well. Body weight was added as an allometric function on all clearance and volume parameters. A statistically significant decrease in estimated terminal piperaquine half-life in pregnant compared with non-pregnant women was found, but there were no differences in post-hoc estimates of total piperaquine exposure. The MCMP analysis indicated a minimum of 13 pregnant and 13 non-pregnant women were required to identify pregnancy as a covariate on relevant pharmacokinetic parameters $(80 \%$ power and $\mathrm{p}=0.05)$. Pregnancy was, therefore, evaluated as a categorical and continuous covariate (i.e. estimate gestational age) in a full covariate approach. Using this approach pregnancy was not associated with any major change in piperaquine elimination clearance. However, a trend of increasing elimination clearance with increasing gestational age could be seen.

(Continued on next page)
\end{abstract}

\footnotetext{
* Correspondence: joel@tropmedres.ac

'Deceased

${ }^{3}$ Mahidol-Oxford Tropical Medicine Research Unit, Faculty of Tropical Medicine, Mahidol University, 60th Anniversary Chalermprakiat Building, 420/6 Rajvithi Road, Bangkok 10400, Thailand

${ }^{4}$ Centre for Tropical Medicine, Department of Clinical Medicine, Churchill Hospital, Oxford, UK

Full list of author information is available at the end of the article
} 


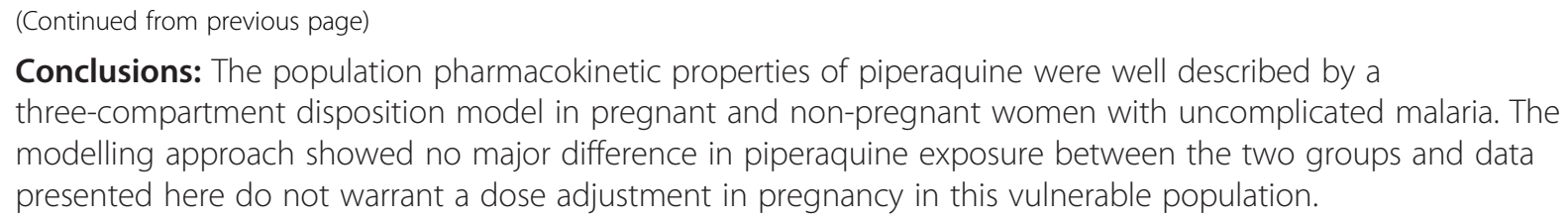

Keywords: Malaria, Piperaquine, Pregnancy, Population pharmacokinetics, Nonlinear mixed-effects modelling

\section{Background}

Malaria is one of the most important infectious diseases with an estimated 216 million people infected worldwide in 2010 [1]. Pregnant women are at an increased risk of developing a malaria infection [2] and are at a higher risk of progressing to severe malaria [3-5]. Plasmodium falciparum malaria is a major contributor to maternal mortality in Sudan; around 37\% of all maternal deaths between 1985 and 1999 at the Medani Teaching Hospital in Medani City, Sudan, were attributed to malaria [6]. Malaria also has severe effects on the foetus causing both foetal loss and low birth weight.

Artemisinin-based combination therapy (ACT) is recommended as first-line treatment for $P$. falciparum malaria in all endemic areas. The artemisinin derivatives have a very rapid parasiticidal effect, which substantially reduces the parasite biomass during the first days of treatment. These drugs have a short terminal elimination half-life and are, therefore, used in combination with longer acting anti-malarials, with the aim of preventing recrudescence by killing residual parasites. Combination therapies consisting of drugs with different mechanisms of action also reduce the risk of the development of drug resistance $[7,8]$.

The oral fixed-dose combination of dihydroartemisinin and piperaquine has shown excellent efficacy in the treatment of $P$. falciparum malaria [9-13]. Piperaquine is highly bound to plasma proteins ( $>99.9 \%)$, has a large apparent volume of distribution, (103-874 L/ kg), a low apparent elimination clearance $(0.6-1.3 \mathrm{~L} / \mathrm{h} / \mathrm{kg})$ and, therefore, a long terminal elimination half-life (12-28 days) [14-20].

Pregnancy has considerable effects on the pharmacokinetic properties of many drugs. Renal elimination, expression of metabolising enzymes, volume of body water and the degree of plasma protein binding all change during pregnancy [21-23]. This could result in lower drug plasma concentrations [24]. Previously published studies have reported a decrease in drug exposure during the later stages of pregnancy for artesunate, artemether, dihydroartemisinin, lumefantrine, sulphadoxine, atovaquone, proguanil and cycloguanil [19,25-31]. Other anti-malarial drugs (e.g. quinine and amodiaquine/ desethylamodiaquine) show no differences in pharmacokinetic properties in pregnant women compared to nonpregnant women [32-34].
Only one previous study has investigated the impact of pregnancy on the pharmacokinetic properties of piperaquine in patients with uncomplicated $P$. falciparum malaria [19]. Pregnancy was found to affect the elimination clearance and the bioavailability of piperaquine, but with no change in total drug exposure. This was further supported by a non-compartmental analysis of the same study [35]. No published information is available on the population pharmacokinetic properties of piperaquine in pregnant or non-pregnant women in an African country.

The aim of this study was to describe the population pharmacokinetic properties of piperaquine in pregnant and non-pregnant women with uncomplicated $P$. falciparum malaria in Sudan.

\section{Methods}

\section{Study design}

The study was conducted at the New Halfa Teaching Hospital in New Halfa, Sudan. Clinical details and noncompartmental analysis results are reported in full elsewhere [36]. The participating women received a written and oral explanation of the study in their own language. If the woman could not read, the explanation was read to her. Ethics approval for the study was given by the College of Medical and Technical Studies, Khartoum, Sudan.

Symptomatic pregnant women with uncomplicated $P$. falciparum malaria in their second or third trimester attending the antenatal clinic in New Halfa were eligible to participate in the study. Non-pregnant women with uncomplicated $P$. falciparum malaria were also recruited as controls.

\section{Drug regimen}

All patients received dihydroartemisinin-piperaquine tetra-phosphate (Duo Cotecxin, $40 \mathrm{mg} / 320 \mathrm{mg}$ tablets, Beijing Holley-Cotec Pharmaceuticals, Co., Ltd.) once daily for three days. Drug administration was directly observed and taken with a glass of water under fasting conditions. The number of tablets was based on the patient's body weight to achieve a daily dose of $20 \mathrm{mg}$ piperaquine tetra-phosphate $/ \mathrm{kg}$.

Blood samples were obtained by venous puncture or a three-way tap. PCR, haematology and biochemistry samples were drawn before the first dose and on day 14 . 
Blood samples $(2 \mathrm{~mL})$ for pharmacokinetic analysis were drawn pre-dose and at $1.5,4,8,24,25.5,28,32,48,49$, $50,52,56,60,72 \mathrm{~h}$ after the first dose and on days 5,7 , $14,21,28,35,42,49,56,63$ and 90. The actual time of dosing and sampling were noted and used in the pharmacokinetic analysis. Blood samples were centrifuged at $2000 \times \mathrm{g}$ for 10 minutes and plasma samples stored in liquid nitrogen until the samples were transferred to Khartoum there they were stored in $-80^{\circ} \mathrm{C}$.

\section{Drug analysis}

The chemical analysis was performed using a previously published method with separation and quantification by liquid chromatography (LC) and tandem mass spectrometry (MS/MS) detection [37]. The LC-system was an Agilent 1200 system consisting of a binary LC pump, a vacuum degasser, autosampler and a column compartment. The MS-system was an API 5000 triple/quadruple mass spectrometer with a Turbo $\mathrm{V}$ ionization source. The lower limit of quantification (LLOQ) was set to 1.5 $\mathrm{ng} / \mathrm{mL}$ and the lower limit of detection (LLOD) was set to $0.38 \mathrm{ng} / \mathrm{mL}$. This method reported an intra- and inter-day precision of below $10 \%$ for all quality control samples.

\section{Pharmacokinetic and statistical analysis}

The data were analysed using nonlinear mixed-effects modelling as implemented in NONMEM version VI (Icon Development Solutions, Ellicott City, Maryland, USA) [38]. Piperaquine plasma concentrations were transformed into their natural logarithms to increase the stability of the numeric analysis. Models were fitted to the data using the first-order conditional estimation (FOCE) method with interaction [39-41]. Census, version 1.1 [42], and Xpose, version 4.04 [43], library for $\mathrm{R}$ was used for model diagnostics. Perl-speaks-NONMEM (PsN), version 3.4.2, [44] was used to automate the modelling process and for model diagnostics.

Model discrimination was based the on the objective function value (OFV) computed by NONMEM as $-2 \times \log$ likelihood [45]. The OFV is approximately $x^{2}$ distributed and a decrease in OFV of 3.84 and 6.64 is considered a significant drop with $\mathrm{p}<0.05$ and $\mathrm{p}<0.01$, respectively, when adding one additional parameter (one degree of freedom between two nested models).

Structural models with one-, two-, three- and fourdisposition compartments were fitted to the data. Several alternative absorption models were investigated; firstorder absorption, first-order absorption with lag time, zero-order absorption, sequential zero- and first-order absorption, sequential zero- and first-order absorption with lag time, parallel first- and zero-order absorption, parallel first-order absorption and transit compartment absorption with a fixed number of 1-10 transit compartments. The full implementation of the transit compartment absorption model, which allows the number of transit compartments to vary between patients, was also evaluated [46].

It was assumed that drug elimination took place from the central compartment and the base model was parameterized as elimination clearance, central volume of distribution, inter-compartmental clearance(s) and peripheral distribution volume(s). Bioavailability was added to the model and the population value was fixed to $100 \%$.

The distribution of the individual parameters was assumed to be log-normal and between-subject variability (BSV) was investigated on all parameters as an exponential random effect [Equation 1].

$$
P_{i}=\theta_{p} \cdot e^{\eta_{i, P}}
$$

where $P_{i}$ is the individual estimate for a model parameter (e.g. individual drug clearance) in the $i^{\text {th }}$ individual. $\theta_{p}$ is the population mean of parameter $P$ and $\eta_{i, P}$ is individual $i^{\text {th }}$ deviation from the population mean. BSV is estimated from a normal distribution with variance $\omega^{2}$ and zero mean. Between dose occasion variability (BOV) was evaluated on absorption parameters [Equation 2].

$$
P_{i j}=\theta_{P} \cdot e^{\left(\eta_{i, P}+\kappa_{j, P}\right)}
$$

Where $\mathrm{P}_{\mathrm{ij}}$ is the individual parameter estimate for the $\mathrm{i}^{\text {th }}$ patient on the $\mathrm{j}^{\text {th }}$ dose occasion, $\mathrm{k}$ is the deviation from the population mean after each dose occasion, taken from a normal distribution with variance $\Pi^{2}$ and zero mean. An additive residual error model was assumed since data were transformed into their natural logarithms (i.e. essentially equivalent to an exponential error model on an arithmetic scale). Body weight was tried in the model as a simultaneous incorporation of an allometric function on all clearance (power of 0.75) and volume parameters (power of 1), considering the strong biological prior of this covariate relationship [47-49].

Basic goodness-of-fit plots and simulation-based diagnostics were used to evaluate the final model. Visual and numerical predictive checks [50] were performed using 2000 simulations at each concentration-time point with binning based on protocol times. The $5^{\text {th }}, 50^{\text {th }}$ and $95^{\text {th }}$ percentile of observed data were plotted over the simulated $95 \%$ confidence interval of the same percentiles to evaluate the models predictive performance. Bootstrap diagnostics were performed using 1000 re-sampled datasets, stratified on pregnancy.

A Monte Carlo Mapped Power analysis (MCMP) [51] was conducted based on results from a previously published population analysis [19]. The current study design in terms of sampling times and doses were used to create a modelled data set with 408 pregnant and 408 non- 
pregnant patients. This data set and the published population pharmacokinetic model was used to estimate the minimum number of individuals needed in each group (i.e. pregnant and non-pregnant) to identify the described covariate relationships (i.e. a $45.0 \%$ increase in elimination clearance or a $46.8 \%$ increase in bioavailability) at a given power (80\%) and significance level $(\mathrm{p}=0.05)$. Full power curves were produced by plotting number of patients needed against the power to detect the assumed covariate relationship.

Pregnancy was investigated in the final pharmacokinetic model utilizing a full covariate approach where pregnancy was included as a categorical covariate on all pharmacokinetic parameters. Estimated gestational age (EGA) was evaluated as a continuous covariate on individual parameters using a linear and a power function, and the most appropriate covariate relationship (lowest OFV) was incorporated into a full covariate model for EGA. These two full covariate models were bootstrapped $(n=200)$ to investigate the impact of pregnancy. A pregnancy related change in the parameter estimate of more than $20 \%$ was deemed to have clinical relevance.

\section{Results}

Fourteen non-pregnant and twelve pregnant women were recruited into the study but two non-pregnant women withdrew their consent and were excluded from the analysis. Full demographics are given in Table 1. The treatment was well-tolerated, none of the patients vomited after treatment and no severe adverse events were reported during the study. One non-pregnant woman had a PCR-confirmed new infection on day 35. None of the women presented recrudescent malaria during the nine weeks of follow-up.

Five hundred sixty-four (564) post-dose plasma samples of piperaquine were used in the pharmacokinetic analysis. A total of $7(1.2 \%)$ of these samples were measured to be below the limit of quantification and omitted. A threecompartmental disposition model resulted in a significantly better model fit compared with a two-compartment model $(\triangle \mathrm{OFV}=-32.8)$. An additional peripheral compartment (four-compartment disposition model) did not further improve the fit $(\triangle \mathrm{OFV}=0)$. A transitcompartment $(\mathrm{n}=3)$ absorption model was superior to all other absorption models $(\triangle \mathrm{OFV} \geq-44.5)$. In the final model the absorption rate constant $\left(\mathrm{k}_{\mathrm{a}}\right)$ and the transit-compartment rate constant $\left(\mathrm{k}_{\mathrm{tr}}\right)$ were set equal to increase the stability of the model. An additive residual error model was adequate in describing the residual random variability. BSV could be estimated reliably on elimination clearance, one intercompartmental clearance parameter, and one peripheral volume parameter. The addition of BSV on the bioavailability resulted in a significant improvement in model fit $(\triangle \mathrm{OFV}=-70.9)$. BOV had considerable impact on both mean transit absorption time and bioavailability $(\triangle \mathrm{OFV}=-340)$. The final parameter estimates and a schematic picture of the final structural model is presented in Table 2 and Figure 1. Body weight was incorporated with an allometric function on all clearance and volume parameters $(\triangle \mathrm{OFV}=-6.14)$.

Secondary parameters (i.e. total drug exposure, maximum concentration after first dose, time to maximum concentration, elimination half-life and day 7 concentrations) were analysed using the Mann-Whitney U-test to

Table 1 Admission demographic data of study population

\begin{tabular}{|c|c|c|c|}
\hline & Non-pregnant women & Pregnant women & P-value \\
\hline & Median (Range) & Median (Range) & \\
\hline Number of patients & 12 & 12 & - \\
\hline Daily piperaquine (phosphate) dose (mg/kg) & $18.1(15.1-24.2)$ & $17.6(13.6-21.7)$ & 0.884 \\
\hline Daily piperaquine (base) dose (mg/kg) & $10.5(8.71-13.9)$ & $10.2(7.83-12.5)$ & 0.884 \\
\hline Age (years) & $21.0(16.0-43.0)$ & $26.0(18.0-33.0)$ & 0.977 \\
\hline Body weight (kg) & $53.0(44.0-81.0)$ & $59.0(50.0-72.0)$ & 0.544 \\
\hline Height $(\mathrm{cm})$ & $163(150-174)$ & $166(150-174)$ & 0.908 \\
\hline Estimated gestational age (weeks) & - & $32.0(15.3-40.1)$ & - \\
\hline Parasitemia (parasites $/ \mu \mathrm{L}$ ) & $13200(936-68700)$ & $12900(624-118000)$ & 0.488 \\
\hline Days of fever & $2.5(1-6)$ & $3(1-6)$ & 0.095 \\
\hline Fever $\left({ }^{\circ} \mathrm{C}\right)$ & $38.3(36.7-40.0)$ & $38.2(36.6-39.9)$ & 0.795 \\
\hline Haemoglobin $(\mathrm{g} / \mathrm{dL})$ & $8.70(7.60-11.5)$ & $9.65(8.00-12.0)$ & 0.099 \\
\hline Urea $(\mathrm{mg} / \mathrm{dL})$ & $26.0(24.0-28.0)$ & $25.0(24.0-28.0)$ & 0.036 \\
\hline Serum glutamic pyruvic transaminase (IU/L) & $5.00(2.00-10.0)$ & $4.50(2.00-9.00)$ & 0.445 \\
\hline Serum glutamic oxaloacetic transaminase (IU/L) & $13.5(2.00-18.0)$ & $13.0(3.00-21.0)$ & 0.727 \\
\hline
\end{tabular}

The $p$-values are calculated using the Mann-Whitney U-test. 
Table 2 Final parameter estimates describing the piperaquine population pharmacokinetics in women with uncomplicated $P$. falciparum malaria

\begin{tabular}{|c|c|c|c|c|}
\hline & Population estimates [RSE \%] & $95 \% \mathrm{Cl}$ & BSV/BOV† [RSE \%] & $95 \% \mathrm{Cl}$ \\
\hline $\mathrm{CL} / \mathrm{F}(\mathrm{L} / \mathrm{h})$ & $44.6[9.90]$ & $37.3-53.8$ & $22.5[31.5]$ & $14.6-29.0$ \\
\hline$V_{C} / F(L)$ & $1820[11.5]$ & $1450-2240$ & - & - \\
\hline $\mathrm{Q}_{1} / \mathrm{F}(\mathrm{L} / \mathrm{h})$ & $47.7[19.0]$ & $32.4-69.2$ & - & - \\
\hline$V_{P_{1}} / F(L)$ & $15900[12.3]$ & $12600-20400$ & - & - \\
\hline $\mathrm{Q}_{2} / \mathrm{F}(\mathrm{L} / \mathrm{h})$ & $352[11.1]$ & $283-431$ & - & - \\
\hline$\overline{V_{P 2} / F(L)}$ & $7520[17.1]$ & $5520-10500$ & - & - \\
\hline $\mathrm{MTT}(\mathrm{h})$ & $1.70[8.05]$ & $1.45-2.00$ & $60.7[22.8]+$ & $44.5-76.7$ \\
\hline RUV & $0.0973[5.90]$ & $0.0753-0.120$ & - & - \\
\hline No. of trans comp & $3 f i x$ & - & - & - \\
\hline $\mathrm{F}(\%)$ & $100 \mathrm{fix}$ & - & $\begin{array}{l}34.7[59.2] \\
64.8[15.2] \dagger\end{array}$ & $\begin{array}{l}9.52-54.9 \\
52.8-76.0\end{array}$ \\
\hline
\end{tabular}

Coefficient of variation (\%CV) for between-subject variability (BSV) and ( $\dagger$ ) between occasion variability (BOV) were calculated as the [exp(estimated variance)-1] ${ }^{1 / 2}$. Relative standard errors (RSE) and the $95 \%$ confidence intervals (Cl) were based on 868 successful stratified bootstrap runs (out of 1000) and presented as $100 \times$ (standard deviation/mean value) and as 2.5 to 97.5 percentiles, respectively.

$\mathrm{CL} / \mathrm{F}$ is the apparent elimination clearance. Vc/F is the apparent volume of distribution of the central compartment. Q1/F and Q2/F is the inter-compartment clearance between the central and first and second peripheral compartment, respectively. VP1/F and VP2/F is the apparent volume of distribution of first and second peripheral compartment, respectively. MTT is the mean transit time of the absorption model. RUV is the variance of the residual variability. No. of trans comp is the number of transit compartments used in the absorption phase. $\mathrm{F}$ represents the relative bioavailability.

identify differences between pregnant and non-pregnant women (Table 3). There was a significant difference in terminal elimination half-life $(\mathrm{p}=0.0014)$, time to maximum concentration $(\mathrm{p}=0.0177)$ and maximum concentration $(\mathrm{p}=0.0205)$ [median (range) in pregnant vs. non-pregnant women: 22.1 (19.1-25.8) vs. 25.7 (20.9-33.3) days, 3.07 (1.65-4.64) vs. 1.48 (0.887-4.18) hours and 185 (109-363) vs. $102(40.6-235) \mathrm{ng} / \mathrm{mL}$, respectively]. However, no significant differences were found in day 7 concentrations $(\mathrm{p}=0.67)$, day 28 concentrations $(\mathrm{p}=0.84)$ or the total drug exposure $(\mathrm{p}=0.80)$ between the pregnant and non-pregnant women.
The MCMP analysis (5\% significance level and 80\% power) indicated that $8+8$ and $13+13$ women are needed to detect pregnancy as a covariate on elimination clearance and bioavailability, respectively (Figure 2). A formal stepwise covariate search was therefore not performed since it might result in a biased covariate selection in this small population sample.

Two full covariate models were constructed from the final model to investigate the clinical relevance of pregnancy and EGA separately. Pregnancy had a relatively large impact on mean transit absorption-time, volume of distributions and inter-compartment clearances but no

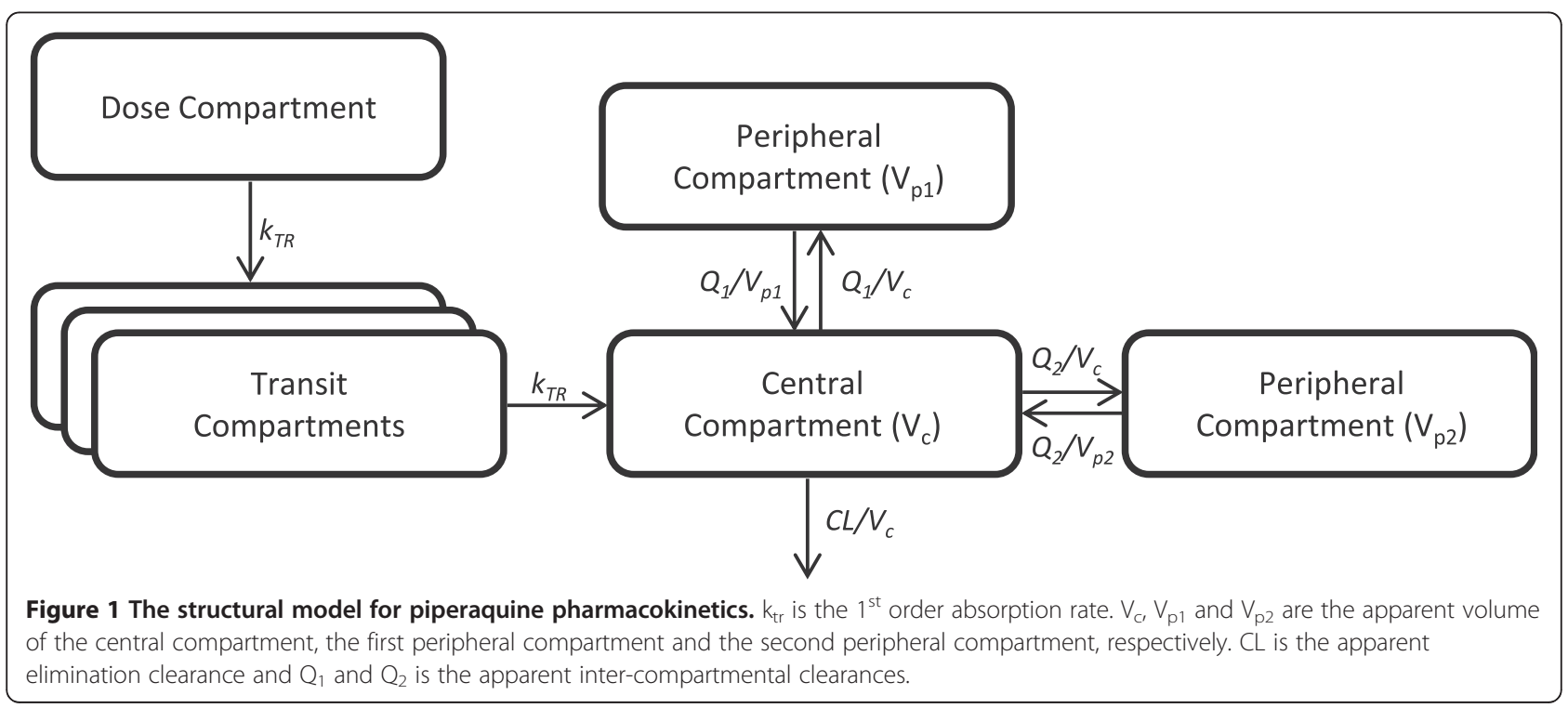


Table 3 Secondary parameters of piperaquine pharmacokinetics in pregnant and non-pregnant women with uncomplicated $P$. falciparum malaria

\begin{tabular}{lllll}
\hline Secondary parameters & Total & Non-pregnant women & Pregnant women & p-value \\
\hline$C_{\text {MAX }}(\mathrm{ng} / \mathrm{mL})$ & $158[40.6-363]$ & $102[40.6-235]$ & $185[109-363]$ & 0.021 \\
\hline$T_{\text {MAX }}$ (hours) & $2.62[0.887-4.64]$ & $1.48[0.887-4.18]$ & $3.07[1.65-4.64]$ & 0.018 \\
\hline Half-life (days) & $23.4[19.1-33.3]$ & $25.7[20.9-33.3]$ & $22.1[19.1-25.8]$ & 0.001 \\
\hline Day 7 concentration $(\mathrm{ng} / \mathrm{mL})$ & $58.3[16.6-146]$ & $55.4[16.6-146]$ & $60.7[40.1-103]$ & 0.671 \\
\hline Day 28 concentration $(\mathrm{ng} / \mathrm{mL})$ & $15.9[4.85-38.6]$ & $15.4[4.85-38.6]$ & $16.1[9.68-26.8]$ & 0.840 \\
\hline AUC $_{0->90}\left(\mathrm{ng}{ }^{*} \mathrm{~h} / \mathrm{mL}\right)$ & $40600[12400-100000]$ & $38000[12400-100000]$ & $42700[27100-68700]$ & 0.799 \\
\hline AUC $_{48 \mathrm{~h}->90}(\mathrm{ng}$ *h/mL) & $36400[10600-90300]$ & $35300[10600-90300]$ & $37700[23500-63200]$
\end{tabular}

Secondary parameters are predicted using the final model and values are presented as median [range]. The p-values are calculated with a Mann-Whitney U-test. $C_{\text {MAX }}$ is the predicted maximum concentration after the first dose and $T_{\text {MAX }}$ is the time to $C_{\text {MAX. }}$. Half-life is the estimated terminal elimination half-life. Day 7 and 28 concentrations are the model predicted plasma concentrations of piperaquine at day 7 and 28 , respectively. AUC $C_{0->90}$ is the predicted area under the concentration-time curve from time zero extrapolated to day $90 . A U C_{48 \mathrm{~h}->90}$ is the predicted area under the concentration-time curve from 48 hours extrapolated to day 90 .

significant effect on elimination clearance (Figure 3). The inclusion of EGA as a power function or a linear function produced similar results. EGA was therefore implemented as a linear function for the full covariate approach and resulted in similar results compared to pregnancy as a categorical covariate (Figure 4A). Bootstrap results for elimination clearance, stratified by trimester (i.e. nonpregnant, second trimester at 20 weeks, and third trimester at 32 weeks) were also investigated and resulted in a non-significant trend of increasing clearance with increasing EGA (Figure 4B).

The final model resulted in good model diagnostic performance and reliable parameter estimates (Figure 5 and Table 2). Calculated epsilon-shrinkage was low (13.0\%) which indicates that model diagnostics can be assessed reliably. However, eta-shrinkage was relatively high for certain parameters (CL/F=18.9\%,
MTT=13.2-52.8\%, F=12.4-46.6\%) (Table 2) and empirical Bayes estimates should therefore be interpreted with caution (Table 3) [52]. The final model had good predictive performance (Figure 6) with 4.8\% (95\% CI. $1.4 \%-11 \%)$ of observed data below and $2.1 \%$ (95\% CI. $1.4 \%-10 \%)$ of observed data above the simulated $90 \%$ prediction interval (the three observations at day 90 were excluded because too few patients were followed up to this time for reliable simulations).

\section{Discussion}

In this study, the pharmacokinetic properties of piperaquine have been investigated using nonlinear mixedeffects modelling in pregnant and non-pregnant Sudanese women treated with piperaquine-dihydroartemisinin for uncomplicated $P$. falciparum malaria. Few studies have been performed to evaluate the effect of pregnancy on

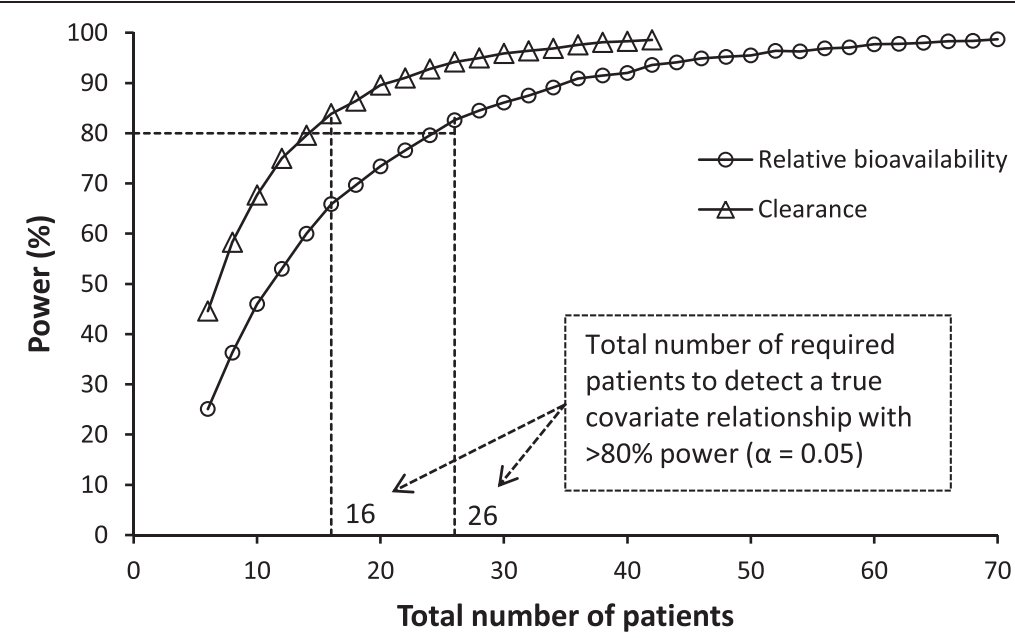

Figure 2 Monte-Carlo Mapped Power (MCMP) curve for identifying pregnancy as a covariate. Triangles represents the power curve for indentifying pregnancy as covariate on apparent elimination clearance and circles is the power curve for identifying pregnancy as a covariate on the relative bioavailability. The dotted black line represents $80 \%$ power. The inserted numbers are the total number of subjects needed to identify pregnancy as a covariate given the used model and study sampling procedure. 


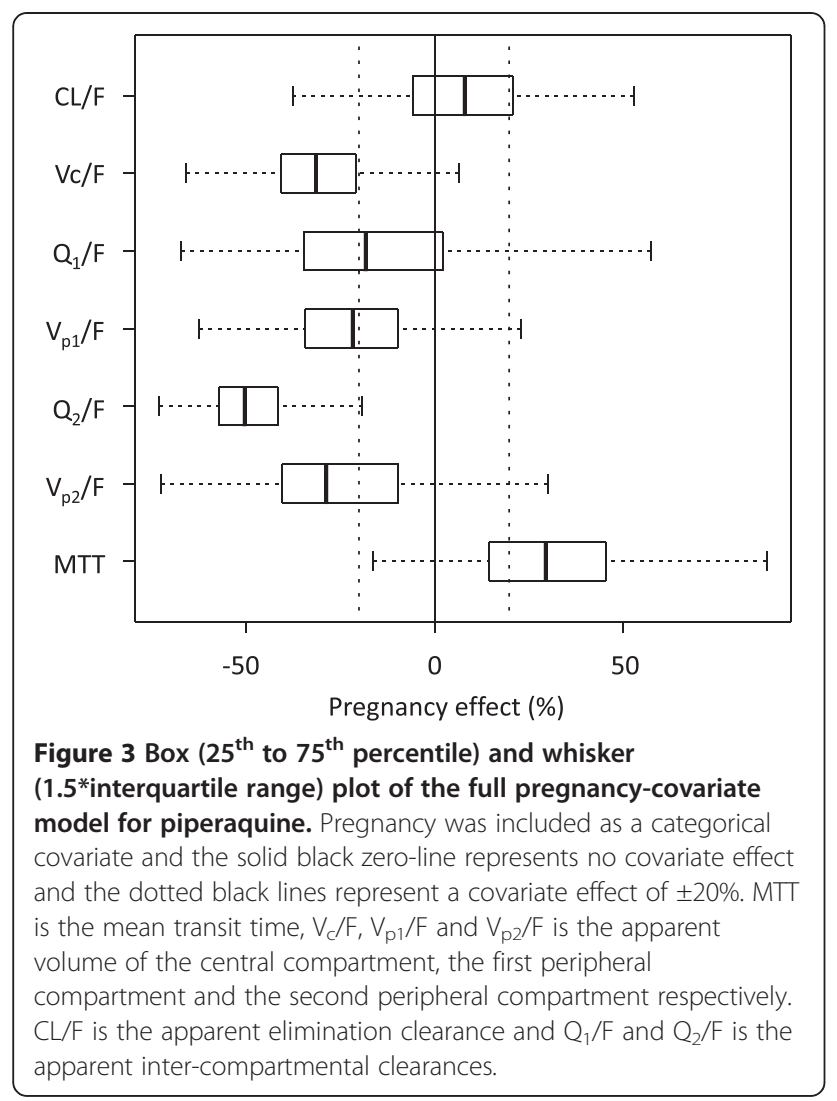

the population pharmacokinetics of piperaquine, and this is the first study conducted in an African population. The treatment was well-tolerated and none of the participating women had recrudescent malaria infections.

Previous studies of piperaquine pharmacokinetics have presented both two- and three-compartment disposition models depending on the amount of data included in the analysis [14,15,17,19,20,53]. A three-compartment disposition model described the piperaquine concentration-time data adequately in this study. This supports the general finding that a three-compartment disposition is more appropriate than a two-compartment disposition when modelling data from patients followed for a sufficient period of time.

The absorption phase was best described with a transit compartment model with three transit compartments including random effects on bioavailability (BSV and $\mathrm{BOV})$ and mean transit absorption time (BOV). The transit-compartment model provides a more physiological representation of the absorption process compared to the absorption models used in previous studies (i.e. first-order absorption and parallel first-order absorption with lag time) [15,17]. Recently published studies have also implemented the transit-compartment model but with two- and five-transit compartments which support the absorption model presented here $[19,20]$. Small variations in the number of transit compartments are to be expected when modelling different

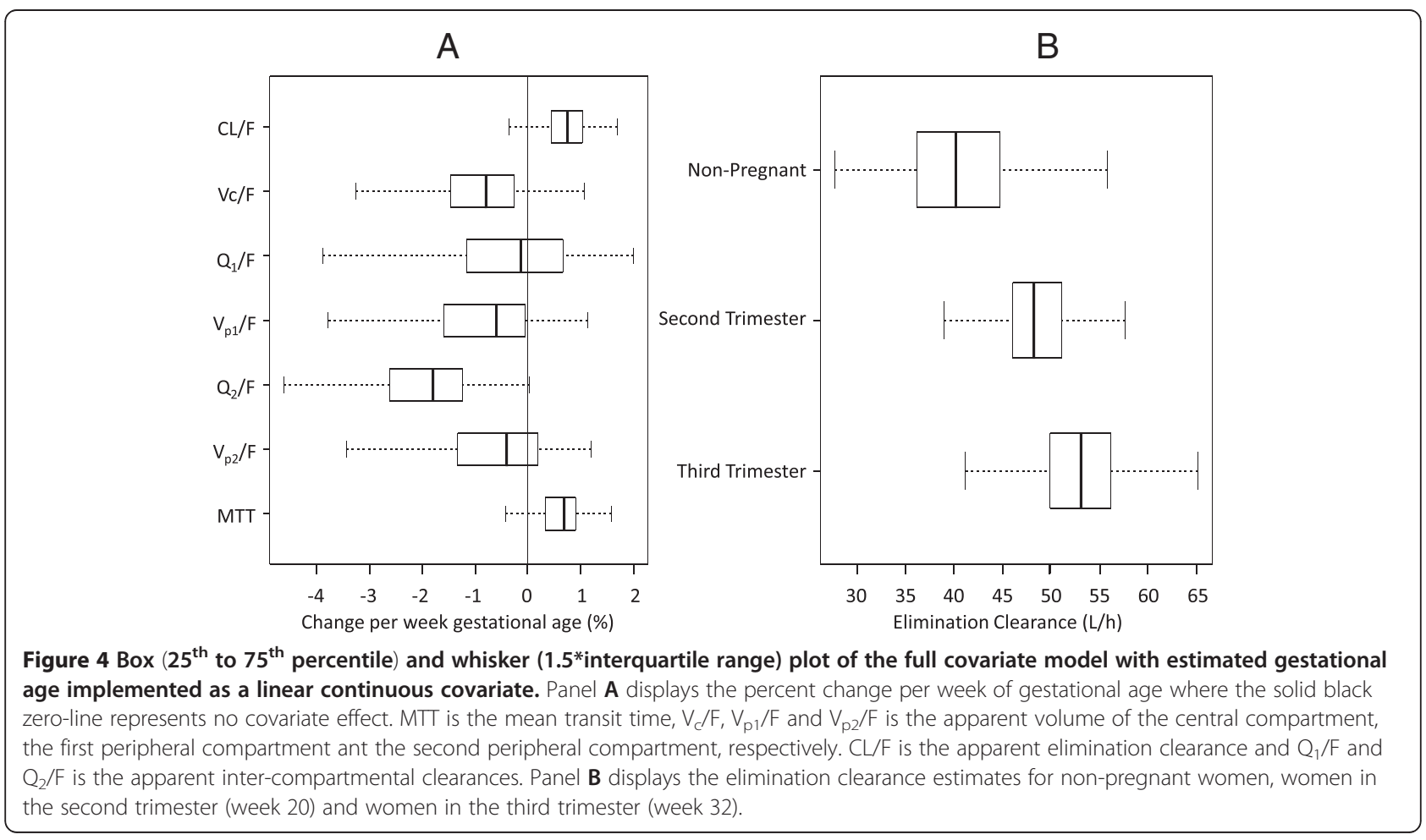



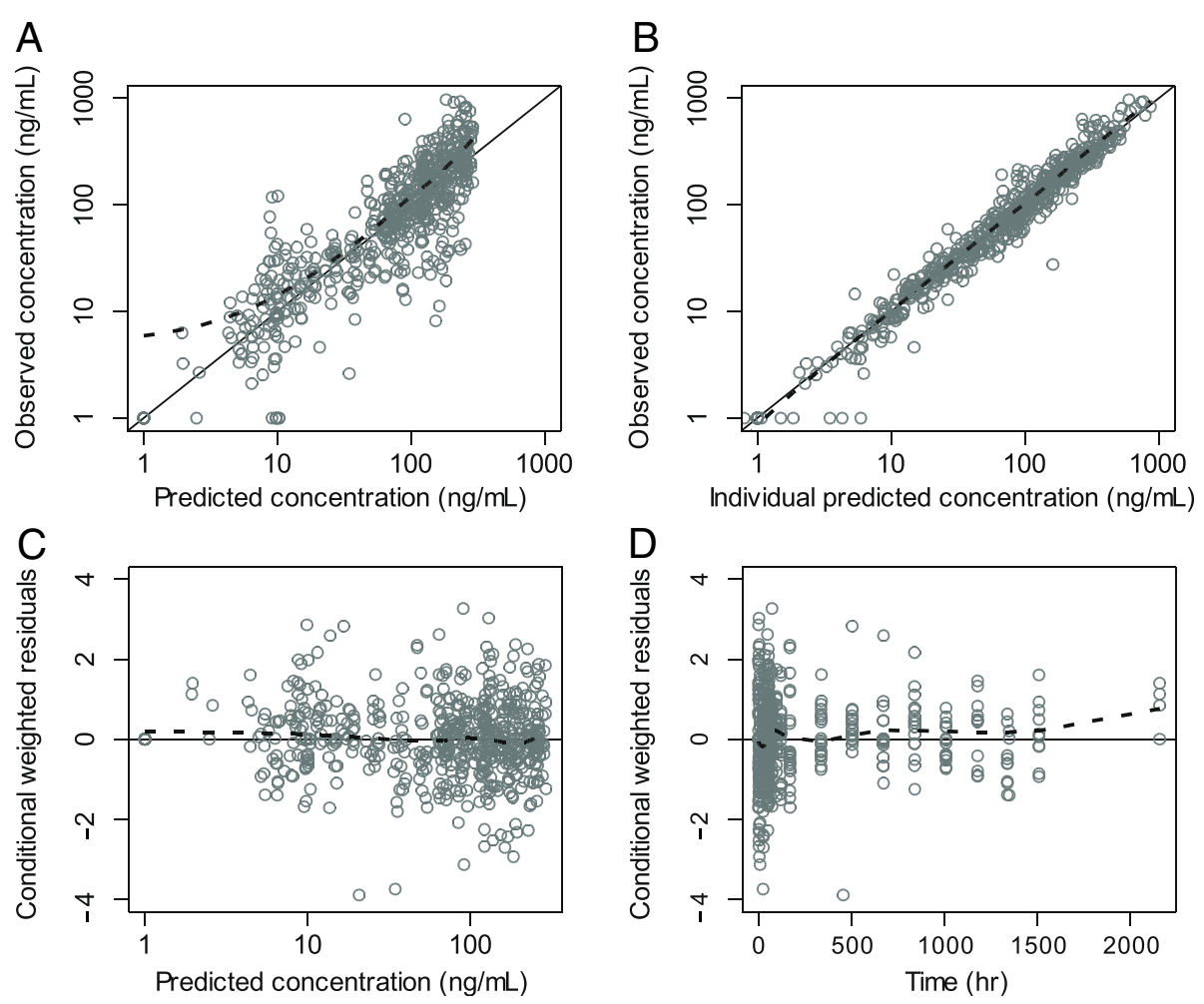

Figure 5 Basic goodness-of-fit plots for the final piperaquine model. Observations plotted against population predicted concentrations (A) and against individual predicted concentrations (B). Conditional weighted residuals plotted against population predicted concentrations (C) and time (D). The solid line is the identity line and the broken line is the locally weighted least square regression line. The concentrations were transformed into their logarithms (base 10). Conditional weighted residuals were fixed between 4 and -4 , excluding one data point.

studies due to population differences, sampling schedules and study size. The inclusion of BSV and BOV in the absorption model improved the description of the data in the absorption phase and accommodated the large between-subject and between-occasion variability in these data. The data in the absorption phase was not rich enough to estimate separate absorption rates for $k_{\mathrm{a}}$ and $\mathrm{k}_{\mathrm{t}}$ and they were therefore set to be equal. This is a common limitation and the same approach has been used in previous studies $[19,20]$. Incorporating BOV resulted in an increasing median bioavailability (0.77, 1.19 and 1.40 at dose 1,2 and 3 , respectively) and mean transit absorption time $(1.55,1.95$ and 2.05 at dose 1,2 and 3, respectively) during the treatment regimen. Similar patterns have been identified in previously studies on piperaquine [19]. This might be an effect of disease recovery or differences in the food intake over the course of the dose regimen. This cannot be verified since parasite densities were not counted at each dose and food intake was not monitored in this study.

Pregnant women had a shorter terminal half-life compared to non-pregnant women, which is in agreement with the non-compartmental analysis [36], and higher maximum concentrations after the first dose. However, there were no differences in total piperaquine exposure, day 7 concentrations or day 28 concentrations, which supports previously published findings in an Asian pregnant population [19].

The main aim of this study was to investigate the pharmacokinetic differences between pregnant and non-pregnant women, but the sample size was not large enough to make a conventional covariate search. The MCMP analysis resulted in a minimum of 13 patients needed in each group in order to identify the previously described covariate relationships with $80 \%$ power and a significance level of 0.05 (Figure 2). However, this is under the assumption of perfect sampling since the MCMP analysis was based on simulated data using protocol sampling times. In the present study, some patients were not sampled for the complete followperiod and some samples were randomly missing which might increase the number of patients needed to identify the assumed covariate-relationships. Therefore the final model did not include any covariates except body weight, which has a strong biological prior $[47,48]$ and in addition gave a drop in OFV when 


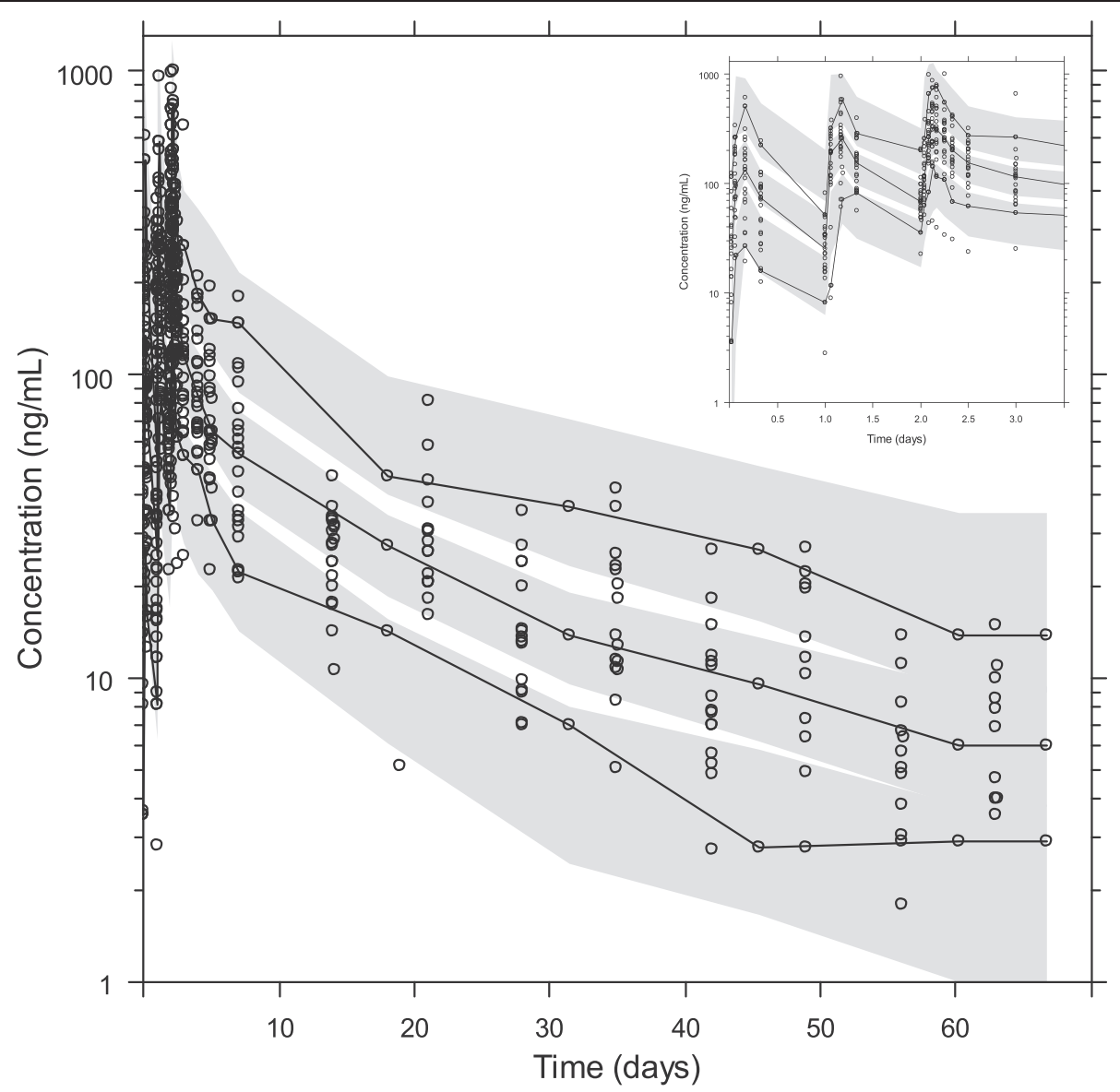

Figure 6 The visual predictive check of the final piperaquine model. The circles represent the observations, the solid line represents the $5^{\text {th }}$, $50^{\text {th }}$ and $95^{\text {th }}$ percentile of the observed data. The shaded areas represent the simulated $95 \%$ confidence interval around the $5^{\text {th }}, 50^{\text {th }}$ and $95^{\text {th }}$ percentile. Concentrations were transformed to their logarithms (base 10). Predicted performance during the three first days is inserted in the top right corner.

included in the model. The full covariate approach suffered from identifiability issues when incorporating the pregnancy covariate simultaneously on clearance parameters, volume parameters and relative bioavailability. The full covariate approach was therefore used to investigate the net effect of a potential covariate on all parameters except relative bioavailability. This approach resulted in a model with reduced volume of distribution and inter-compartment clearance in pregnant women compared with non-pregnant women but no net-effect on apparent clearance. This is in agreement with previously published results where pregnancy affected both clearance and relative bioavailability but in different directions [19]. These covariate relationships would also explain the difference in terminal elimination half-life and the lack of difference in total drug exposure.

The model presented in this study was built on data from few patients and a single individual can therefore have a considerable impact on the results. Piperaquine population pharmacokinetic parameter estimates from the final model are in agreement with previous reports (Table 4). However, the elimination clearance presented in this study for an African population is lower compared to previous studies in non-pregnant and pregnant patients. This might suggest an ethnicity related effect on elimination clearance but this needs to be confirmed in a larger population.

In conclusion, this study presents the population pharmacokinetic properties of piperaquine in pregnant and non-pregnant women with uncomplicated $P$. falciparum malaria in Sudan. The terminal half-life was shorter in pregnant compared to non-pregnant women, but the total drug exposure was comparable between the two groups. This supports previous findings that no dose adjustments are needed on account of altered piperaquine pharmacokinetics in pregnancy. 
Table 4 A literature comparison of the pharmacokinetic properties of piperaquine

\begin{tabular}{|c|c|c|c|c|c|c|c|}
\hline Study population & $\begin{array}{l}\text { Age } \\
\text { (years) }\end{array}$ & $\begin{array}{l}\text { Study size } \\
\text { (Males/Females) }\end{array}$ & $\begin{array}{l}\mathbf{t}_{1 / 2} \\
\text { (days) }\end{array}$ & $\begin{array}{l}\mathrm{CL} / \mathrm{F} \\
(\mathrm{L} / \mathbf{h} / \mathbf{k g})\end{array}$ & $\begin{array}{l}\mathrm{V}_{\mathrm{D}} / \mathrm{F} \\
(\mathrm{L} / \mathbf{k g})\end{array}$ & Method & Reference \\
\hline \multicolumn{8}{|l|}{ Piperaquine pharmacokinetics in pregnant women } \\
\hline Pregnant Sudanese women with uncomplicated $P$. falciparum malaria & 18-33 & $12(0 / 12)$ & 22.1 & 0.678 & 384 & Pop PK & This study \\
\hline $\begin{array}{l}\text { Pregnant Thai and Karen women with uncomplicated } P \text {. falciparum } \\
\text { malaria }\end{array}$ & $18-43$ & $24(0 / 24)$ & 17.5 & 1.28 & 529 & Pop PK & [19] \\
\hline \multicolumn{8}{|l|}{ Piperaquine pharmacokinetics in non-pregnant populations } \\
\hline $\begin{array}{l}\text { Non-pregnant Sudanese women with uncomplicated } P \text {. falciparum } \\
\text { malaria }\end{array}$ & $16-43$ & $12(0 / 12)$ & 25.7 & 0.739 & 446 & Pop PK & This study \\
\hline $\begin{array}{l}\text { Non-pregnant Thai and Karen women with uncomplicated } \\
\text { P. falciparum malaria }\end{array}$ & $18-45$ & $24(0 / 24)$ & 24.0 & 1.32 & 829 & Pop PK & [19] \\
\hline $\begin{array}{l}\text { Non-pregnant Thai and Karen males and females with uncomplicated } \\
\text { P. falciparum malaria }\end{array}$ & $6-52$ & $98(59 / 39)$ & 27.8 & 1.37 & 874 & Pop PK & [17] \\
\hline $\begin{array}{l}\text { Non-pregnant Cambodian males and females with uncomplicated } \\
\text { P. falciparum malaria }\end{array}$ & $30 \pm 13+$ & $38(20 / 18)$ & 22.6 & 0.900 & 574 & Pop PK & [14] \\
\hline Healthy Vietnamese males & $21-45$ & $12(12 / 0)$ & 23.0 & 1.82 & 194 & Pop PK & [15] \\
\hline \multicolumn{8}{|l|}{ Piperaquine pharmacokinetics in children } \\
\hline $\begin{array}{l}\text { Children in Papua New Guinea with uncomplicated P. falciparum } \\
\text { and P. vivax malaria }\end{array}$ & $7.1 \pm 1.5+$ & $12(8 / 4)$ & 21.3 & $0.573^{*}$ & $385^{*}$ & Pop PK & [54] \\
\hline Children in Burkina Faso with uncomplicated $P$. falciparum malaria & $2-10$ & $236(131 / 105)$ & 23.2 & 0.417 & 214 & Pop PK & [20] \\
\hline $\begin{array}{l}\text { Children in Papua New Guinea with uncomplicated P. falciparum, } \\
P \text {. vivax and P. malariae malaria }\end{array}$ & $6.9 \pm 1.4 \dagger$ & $22(17 / 5)$ & 17.2 & 0.850 & 431 & Pop PK & [53] \\
\hline Cambodian children with uncomplicated $P$. falciparum malaria & $7 \pm 2+$ & $47(26 / 21)$ & 13.5 & 1.85 & 614 & Pop PK & [14] \\
\hline \multicolumn{8}{|l|}{ Influence of diet on piperaquine pharmacokinetics } \\
\hline $\begin{array}{l}\text { Fasting non-pregnant Thai and Karen males and females with } \\
\text { uncomplicated P. falciparum malaria }\end{array}$ & $18-55$ & $15(13 / 2)$ & 17.5 & 1.19 & 700 & NCA & [18] \\
\hline $\begin{array}{l}\text { Fed non-pregnant Thai and Karen males and females with } \\
\text { uncomplicated } P \text {. falciparum malaria }\end{array}$ & $19-41$ & $15(13 / 2)$ & 21.4 & 1.01 & 769 & NCA & [18] \\
\hline Healthy Caucasian males and non-pregnant women, low fat meal & $19-42$ & $8(4 / 4)$ & $20.3^{*}$ & $1.14^{*}$ & $716^{*}$ & NCA & [16] \\
\hline Healthy Caucasian males and non-pregnant women, high fat meal & $19-42$ & $8(4 / 4)$ & $20.9^{*}$ & $0.60^{*}$ & $365^{*}$ & NCA & [16] \\
\hline
\end{tabular}

$\mathrm{CL} / \mathrm{F}$ is the apparent elimination clearance, $t_{1 / 2}$ is the elimination half-life, $V_{D} / F$ is the apparent volume of distribution, Pop PK represents a population pharmacokinetic analysis and NCA represents a non-compartmental analysis. Age is given as a range or ( $\dagger$ ) as mean \pm standard deviation. Other parameters are given as median or as mean when indicated $(*)$.

\section{Competing interests}

The Welcome Trust is a UK-based medical research charity and is independent of all drug companies. It has no financial links with the manufacturers of either the diagnostics tests or the drugs used in this study. The authors declare no conflict of interest.

\section{Authors' contributions}

IA, FN, ND, and NW conceived the project. WH, NL and JT quantified the drug concentrations. RH and JT performed the pharmacokinetic analysis and wrote the first draft of the manuscript. All authors revised the manuscript critically for important intellectual content and approved the final manuscript.

\section{Acknowledgment}

We sincerely thank all women for their participation in completing this study. We also thank the diligent staff from New Halfa Teaching Hospital. The study treatment was kindly provided by Bejing Holley-Cotec Pharmaceuticals, Co., Ltd. (Bejing, China). Drug assays were supported by the Malaria in Pregnancy (MIP) consortium, which is funded through a grant from the Bill and Melinda Gates Foundation to the Liverpool School of Tropical Medicine. This investigation was part of the Wellcome Trust-Mahidol University-Oxford Tropical Medicine Research Programme, and the PKPDia collaboration, both supported by the Wellcome Trust of Great Britain.

\section{Author details}

'Unit for Pharmacokinetics and Drug Metabolism, Department of Pharmacology, University of Gothenburg, Gothenburg, Sweden. ${ }^{2}$ Faculty of Medicine, University of Khartoum, Khartoum, Sudan. ${ }^{3}$ Mahidol-Oxford Tropical Medicine Research Unit, Faculty of Tropical Medicine, Mahidol University, 60th Anniversary Chalermprakiat Building, 420/6 Rajvithi Road, Bangkok 10400, Thailand. ${ }^{4}$ Centre for Tropical Medicine, Department of Clinical Medicine, Churchill Hospital, Oxford, UK. ${ }^{5}$ Shoklo Malaria Research Unit, Mae Sot, Thailand.

Received: 20 September 2012 Accepted: 22 November 2012 Published: 29 November 2012

\section{References}

1. WHOS: World malaria report: 2011. Switzerland: World Health Organization; 2011.

2. Lindsay S, Ansell J, Selman C, Cox V, Hamilton K, Walraven G: Effect of pregnancy on exposure to malaria mosquitoes. Lancet 1972, 2000:355.

3. Schantz-Dunn J, Nour NM: Malaria and pregnancy: a global health perspective. Rev Obstet Gynecol 2009, 2:186-192.

4. Abrams ET, Meshnick SR: Malaria during pregnancy in endemic areas: a lens for examining maternal-fetal conflict. Am J Hum Biol 2009, 21:643-650.

5. Smereck J: Malaria in pregnancy: update on emergency management. J Emerg Med 2010, 40:393-396. 
6. Dafallah SE, El-Agib FH, Bushra GO: Maternal mortality in a teaching hospital in Sudan. Saudi Med J 2003, 24:369-372.

7. Nosten F, Brasseur P: Combination therapy for malaria: the way forward? Drugs 2002, 62:1315-1329.

8. White NJ: Delaying antimalarial drug resistance with combination chemotherapy. Parasitologica 1999, 41:301-308.

9. Ashley EA, Krudsood S, Phaiphun L, Srivilairit S, McGready R, Leowattana W Hutagalung R, Wilairatana P, Brockman A, Looareesuwan S, Nosten F, White NJ: Randomized, controlled dose-optimization studies of dihydroartemisinin-piperaquine for the treatment of uncomplicated multidrug-resistant falciparum malaria in Thailand. J Infect Dis 2004, 190:1773-1782.

10. Ashley EA, McGready R, Hutagalung R, Phaiphun L, Slight T, Proux S, Thwai $\mathrm{KL}$, Barends $\mathrm{M}$, Looareesuwan S, White NJ, Nosten F: A randomized, controlled study of a simple, once-daily regimen of dihydroartemisininpiperaquine for the treatment of uncomplicated, multidrug-resistant falciparum malaria. Clin Infect Dis 2005, 41:425-432.

11. Denis MB, Davis TM, Hewitt S, Incardona S, Nimol K, Fandeur T, Poravuth Y Lim C, Socheat D: Efficacy and safety of dihydroartemisinin-piperaquine (Artekin) in Cambodian children and adults with uncomplicated falciparum malaria. Clin Infect Dis 2002, 35:1469-1476.

12. Karunajeewa H, Lim C, Hung TY, Ilett KF, Denis MB, Socheat D, Davis TM: Safety evaluation of fixed combination piperaquine plus dihydroartemisinin (Artekin) in Cambodian children and adults with malaria. Br J Clin Pharmacol 2004, 57:93-99.

13. Tran TH, Dolecek C, Pham PM, Nguyen TD, Nguyen TT, Le HT, Dong TH, Tran T, Stepniewska K, White NJ, Farrar J: Dihydroartemisinin-piperaquine against multidrug-resistant Plasmodium falciparum malaria in Vietnam: randomised clinical trial. Lancet 2004, 363:18-22.

14. Hung TY, Davis TM, llett KF, Karunajeewa H, Hewitt S, Denis MB, Lim C, Socheat D: Population pharmacokinetics of piperaquine in adults and children with uncomplicated falciparum or vivax malaria. Br J Clin Pharmacol 2004, 57:253-262.

15. Roshammar D, Hai TN, Friberg Hietala S, Van Huong N, Ashton M: Pharmacokinetics of piperaquine after repeated oral administration of the antimalarial combination CV8 in 12 healthy male subjects. Eur J Clin Pharmacol 2006, 62:335-341.

16. Sim IK, Davis TM, llett KF: Effects of a high-fat meal on the relative oral bioavailability of piperaquine. Antimicrob Agents Chemother 2005, 49:2407-2411.

17. Tarning J, Ashley EA, Lindegardh N, Stepniewska K, Phaiphun L, Day NP, McGready R, Ashton M, Nosten F, White NJ: Population pharmacokinetics of piperaquine after two different treatment regimens with dihydroartemisinin-piperaquine in patients with Plasmodium falciparum malaria in Thailand. Antimicrob Agents Chemother 2008, 52:1052-1061.

18. Annerberg A, Lwin KM, Lindegardh N, Khrutsawadchai S, Ashley E, Day NP, Singhasivanon P, Tarning J, White NJ, Nosten F: A small amount of fat does not affect piperaquine exposure in patients with malaria. Antimicrob Agents Chemother 2011, 55:3971-3976.

19. Tarning J, Rijken MJ, McGready R, Phyo AP, Hanpithakpong W, Day NP, White NJ, Nosten F, Lindegardh N: Population pharmacokinetics of dihydroartemisinin and piperaquine in pregnant and nonpregnant women with uncomplicated malaria. Antimicrob Agents Chemother 2012, 56:1997-2007.

20. Tarning J, Zongo I, Somé FA, Rouamba N, Parikh S, Rosenthal PJ, Hanpithakpong W, Jongrak N, Day NP, White NJ, Nosten F, Ouedraogo JB, Lindegardh N: Population pharmacokinetics and pharmacodynamics of piperaquine in children with uncomplicated falciparum malaria. Clin Pharmacol Ther 2012, 91:497-505.

21. Dawes $M$, Chowienczyk PJ: Drugs in pregnancy. Pharmacokinetics in pregnancy. Best Pract Res Clin Obstet Gynaecol 2001, 15:819-826.

22. Dean M, Stock B, Patterson RJ, Levy G: Serum protein binding of drugs during and after pregnancy in humans. Clin Pharmacol Ther 1980, 28:253-261.

23. Anderson GD: Pregnancy-induced changes in pharmacokinetics: a mechanistic-based approach. Clin Pharmacokinet 2005, 44:989-1008.

24. McGready R, Tan SO, Ashley EA, Pimanpanarak M, Viladpai-Nguen J, Phaiphun L, Wustefeld K, Barends M, Laochan N, Keereecharoen L, Lindegardh N, Singhasivanon P, White NJ, Nosten F: A randomised controlled trial of artemether-lumefantrine versus artesunate for uncomplicated plasmodium falciparum treatment in pregnancy. PLOS Med 2008, 5:e253.

25. Green MD, van Eijk AM, van Ter Kuile FO, Ayisi JG, Parise ME, Kager PA, Nahlen BL, Steketee R, Nettey H: Pharmacokinetics of sulfadoxine-pyrimethamine in HIV-infected and uninfected pregnant women in Western Kenya. J Infect Dis 2007, 196:1403-1408.

26. McGready R, Ashley EA, Moo E, Cho T, Barends M, Hutagalung R, Looareesuwan S, White NJ, Nosten F: A randomized comparison of artesunate-atovaquone-proguanil versus quinine in treatment for uncomplicated falciparum malaria during pregnancy. J Infect Dis 2005, 192:846-853.

27. McGready R, Stepniewska K, Edstein MD, Cho T, Gilveray G, Looareesuwan S, White NJ, Nosten F: The pharmacokinetics of atovaquone and proguanil in pregnant women with acute falciparum malaria. Eur J Clin Pharmacol 2003, 59:545-552.

28. McGready R, Stepniewska K, Lindegardh N, Ashley EA, La Y, Singhasivanon $P$, White NJ, Nosten F: The pharmacokinetics of artemether and lumefantrine in pregnant women with uncomplicated falciparum malaria. Eur J Clin Pharmacol 2006, 62:1021-1031.

29. McGready R, Stepniewska K, Ward SA, Cho T, Gilveray G, Looareesuwan S, White NJ, Nosten F: Pharmacokinetics of dihydroartemisinin following oral artesunate treatment of pregnant women with acute uncomplicated falciparum malaria. Eur J Clin Pharmacol 2006, 62:367-371.

30. Tarning J, McGready R, Lindegardh N, Ashley EA, Pimanpanarak M, Kamanikom B, Annerberg A, Day NP, Stepniewska K, Singhasivanon P, et al: Population pharmacokinetics of lumefantrine in pregnant women treated with artemether-lumefantrine for uncomplicated Plasmodium falciparum malaria. Antimicrob Agents Chemother 2009, 53:3837-3846.

31. Tarning J, Kloprogge F, Piola P, Dhorda M, Muwanga S, Turyakira E, Nuengchamnong N, Nosten F, Day NP, White NJ, et al: Population pharmacokinetics of Artemether and dihydroartemisinin in pregnant women with uncomplicated Plasmodium falciparum malaria in Uganda. Malar J 2012, 11:293.

32. Abdelrahim II, Adam I, Elghazali G, Gustafsson LL, Elbashir MI, Mirghani RA Pharmacokinetics of quinine and its metabolites in pregnant Sudanese women with uncomplicated Plasmodium falciparum malaria. J Clin Pharm Ther 2007, 32:15-19.

33. Rijken MJ, McGready R, Jullien V, Tarning J, Lindegardh N, Phyo AP, Win AK, Hsi P, Cammas M, Singhasivanon P, White NJ, Nosten F: Pharmacokinetics of amodiaquine and desethylamodiaquine in pregnant and postpartum women with Plasmodium vivax malaria. Antimicrob Agents Chemother 2011, 55:4338-4342.

34. Tarning J, Chotsiri P, Jullien V, Rijken MJ, Bergstrand M, Cammas M, McGready R, Singhasivanon P, Day NP, White NJ, Nosten F, Lindegardh N: Population pharmacokinetic and pharmacodynamic modeling of amodiaquine and desethylamodiaquine in women with Plasmodium vivax malaria during and after pregnancy. Antimicrob Agents Chemother 2012, 56:5764-5773

35. Rijken MJ, McGready R, Phyo AP, Lindegardh N, Tarning J, Laochan N, Than $\mathrm{HH}, \mathrm{Mu} \mathrm{O}$, Win AK, Singhasivanon P, White N, Nosten F: Pharmacokinetics of dihydroartemisinin and piperaquine in pregnant and nonpregnant women with uncomplicated falciparum malaria. Antimicrob Agents Chemother 2011, 55:5500-5506.

36. Adam I, Tarning J, Lindegardh N, Mahgoub H, McGready R, Nosten F: Pharmacokinetics of piperaquine in pregnant women in Sudan with uncomplicated Plasmodium falciparum malaria. AmJTrop Med Hyg 2012, 87:35-40.

37. Lindegardh N, Annerberg A, White NJ, Day NP: Development and validation of a liquid chromatographic-tandem mass spectrometric method for determination of piperaquine in plasma stable isotope labeled internal standard does not always compensate for matrix effects. J Chromatogr B Analyt Technol Biomed Life Sci 2008, 862:227-236.

38. Beal SLLBS, Boeckmann AJ: NONMEM Users Guides. City, Maryland, USA Ellicott City, Maryland, USA; 2006.

39. Wahlby U, Bouw MR, Jonsson EN, Karlsson MO: Assessment of type I error rates for the statistical sub-model in NONMEM. J Pharmacokinet Pharmacodyn 2002, 29:251-269.

40. Wahlby U, Jonsson EN, Karlsson MO: Assessment of actual significance levels for covariate effects in NONMEM. J Pharmacokinet Pharmacodyn 2001, 28:231-252. 
41. Beal AJB SL, Sheiner LB: NONMEM users guides. University of Califonia. San Fransisco, CA: NONMEM project group; 1992.

42. Wilkins Jj: NONMEMory: a run management tool for NONMEM. Comput Methods Programs Biomed 2005, 78:259-267.

43. Jonsson EN, Karlsson MO: Xpose-an S-PLUS based population pharmacokinetic/pharmacodynamic model building aid for NONMEM. Comput Methods Programs Biomed 1999, 58:51-64.

44. Lindbom L, Pihlgren P, Jonsson EN: PsN-Toolkit-a collection of computer intensive statistical methods for non-linear mixed effect modeling using NONMEM. Comput Methods Programs Biomed 2005, 79:241-257.

45. Beal SL, Sheiner LB: Estimating population kinetics. Crit Rev Biomed Eng 1982, 8:195-222.

46. Savic RM, Jonker DM, Kerbusch T, Karlsson MO: Implementation of a transit compartment model for describing drug absorption in pharmacokinetic studies. J Pharmacokinet Pharmacodyn 2007, 34:711-726.

47. Anderson BJ, Holford NH: Mechanism-based concepts of size and maturity in pharmacokinetics. Annu Rev Pharmacol Toxicol 2008, 48:303-332.

48. Anderson $\mathrm{BJ}$, Holford NH: Mechanistic basis of using body size and maturation to predict clearance in humans. Drug Metab Pharmacokinet 2009, 24:25-36.

49. McLeay SC, Morrish GA, Kirkpatrick CM, Green B: The relationship between drug clearance and body size: systematic review and meta-analysis of the literature published from 2000 to 2007. Clin Pharmacokinet 2012 51:319-330

50. Holford N: A degenerative predictive check [abstract]. 14th annual Meeting Population approach group Europe. 2005, 738:14.

51. Vong C, Bergstrand M, Nyberg J, Karlsson MO: Rapid sample size calculations for a defined likelihood ratio test-based power in mixed-effects models. AAPS J 2012, 14:176-186.

52. Savic RM, Karlsson MO: Importance of shrinkage in empirical bayes estimates for diagnostics: problems and solutions. AAPS J 2009, 11:558-569.

53. Karunajeewa HA, llett KF, Mueller I, Siba P, Law I, Page-Sharp M, Lin E, Lammey J, Batty KT, Davis TM: Pharmacokinetics and efficacy of piperaquine and chloroquine in Melanesian children with uncomplicated malaria. Antimicrob Agents Chemother 2008, 52:237-243.

54. Salman S, Page-Sharp M, Batty KT, Kose K, Griffin S, Siba PM, llett KF, Mueller I, Davis TM: Pharmacokinetic comparison of two piperaquine-containing artemisinin combination therapies in Papua New Guinean children with uncomplicated malaria. Antimicrob Agents Chemother 2012, 56:3288-3297.

doi:10.1186/1475-2875-11-398

Cite this article as: Hoglund et al:: A population pharmacokinetic model of piperaquine in pregnant and non-pregnant women with uncomplicated Plasmodium falciparum malaria in Sudan. Malaria Journal 2012 11:398.

\section{Submit your next manuscript to BioMed Central and take full advantage of:}

- Convenient online submission

- Thorough peer review

- No space constraints or color figure charges

- Immediate publication on acceptance

- Inclusion in PubMed, CAS, Scopus and Google Scholar

- Research which is freely available for redistribution 